\title{
REMARK ON THE POINCARE DUALITY THEOREM
}

\author{
WILLIAM BROWDER ${ }^{1}$
}

1. Introduction. In this note we study the homology properties of spaces $X$ in which Poincaré duality holds, such as compact orientable manifolds. For such spaces we show that Poincaré duality holds also for the Bockstein spectral sequences (see [3]). As a consequence we get:

Theorem 1. Let $X$ be a space with Poincaré duality, $\operatorname{dim} X=2 m+1$, $m$ even. Then either (1) $H_{m}(X)=F+T+T$ or (2) $H_{m}(X)=F+T+T$ $+Z_{2}$, where $F$ is free abelian and $T$ is a torsion group. In case (2) $S q^{m}: H^{m+1}\left(X ; Z_{2}\right) \rightarrow H^{2 m+1}\left(X ; Z_{2}\right)$ is not zero, so that the Stiefel-Whitney class $W \neq 0$. If $m=2$ then $H_{2}(X)=F+T+T+Z_{2}$ if and only if $w_{3} \neq 0$.

Theorem 2. Let $X$ be a space with Poincaré duality, $\operatorname{dim} X=2 m+1$, $m$ even, $H^{i}\left(X ; Z_{2}\right)=0$ for $i<m$. Then $H_{m}(X)=F+T+T+Z_{2}$ if and only if $m=2$ and $w_{3} \neq 0$.

Here $H_{*}(X)$ denotes the integral homology of $X ; Z_{p}$ denotes the integers $\bmod p$.

Similar results to these have been obtained by M. Rueff [4] using Seifert's "Linking invariant" [5]. ${ }^{2}$ C. T. C. Wall [7] has also studied a similar situation. Our methods have similarity to theirs, but use of the Bockstein spectral sequence instead of more conventional homology theories allows us to refine the results somewhat, bringing in Steenrod squares.

I am endebted to Wall for his comments, and to Emery Thomas who pointed out Lemma 7, enabling me to sharpen my original version of Theorem 2.

2. Poincaré duality and the Bockstein spectral sequence. All spaces $X$ considered shall be assumed to have $H_{i}(X)$ finitely generated for all $i$.

Definition. A space $X$ is said to satisfy Poincaré duality if $H_{n}(X)=Z, H_{i}(X)=0$ for $i>n$, and if $\mu \in H_{n}(X)$ is a generator, then $\cap \mu: H^{q}(X) \rightarrow H_{n-q}(X)$ is an isomorphism for all $q$. We will write $n=\operatorname{dim} X$.

We refer to $[3$, p. 48] for the definition of cap product. Let

Presented to the Society, October 28, 1961; received by the editors September 18, 1961.

${ }^{1}$ The author was supported by an NSF grant, NSF G-15984.

2I am endebted to J. Eells and W. Massey for these references. 
$e \in C_{n}(X)$ be a cycle representing $\mu$. Then $\cap e$ is a chain map (see $[3 ;$ Lemma 7.5$])$ and $(\cap e)_{*}=\cap \mu$. (This requires a slight modification of the coboundary.)

We recall that the Bockstein spectral sequence of a chain complex $C$ is the spectral sequence associated with the homology exact couple:

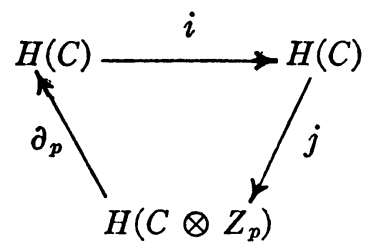

We refer to [3] for details of the definition and properties of the spectral sequence. In particular, a chain map induces a map of spectral sequences, and a chain map which induces homology isomorphism induces isomorphism of spectral sequences.

Hence, we may consider $\cap \mu$ as inducing isomorphism of the cohomology Bockstein spectral sequence in dimension $q,\left(E_{(r)}^{q}\right)$, with the homology Bockstein spectral sequence in dimension $n-q,\left(E_{n-q}^{(r)}\right)$. By the usual argument, this is equivalent to the statement that the cup product pairing $E_{(r)}^{q} \otimes E_{(r)}^{n-q} \rightarrow E_{(r)}^{n}=Z_{p}$ is a nonsingular pairing.

LEMMA 1. $\operatorname{dim}\left(\text { image } d_{r}\right)^{q}=$ number of summands $Z_{p} r$ in $H^{q}(C)$, $\left(d_{r}=\right.$ the differential in the cohomology Bockstein spectral sequence $\left.E_{(r)}\right)$.

This lemma follows immediately from the results of $[3, \S 3]$.

Lemma 2. If $X$ satisfies Poincaré duality and $\operatorname{dim} X=n$, then (image $\left.d_{r}\right)^{n}=0$.

This follows immediately from Lemma 1, and the fact that $H^{n}(X)$ $=Z$ is free.

Lemma 3. Let $X$ satisfy Poincaré duality, $\operatorname{dim} X=2 m+1$. If $x, y \in E_{(r)}^{m}$, then $\left(d_{r} x\right) y=(-1)^{m+1} x\left(d_{r} y\right)$.

Proof. $x y \in E_{(r)}^{2 m}$ so that $d_{r}(x y)=\left(d_{r} x\right) y+(-1)^{m} x\left(d_{r} y\right)$, which equals zero by Lemma 2, which proves Lemma 3.

Let $K_{r}=$ kernel of $d_{r}$ in $E_{(r)}^{m}, I_{r}=$ image of $d_{r}$ in $E_{(r)}^{m+1}$. Then $I_{r}$ is orthogonal to $K_{r}$ under the cup product pairing, from Lemma 3 . Hence the cup product induces a nonsingular pairing of $E_{(r)}^{m} / K_{r}$ with $I_{r}$. Further $d_{r}$ induces an isomorphism of $E_{(r)}^{m} / K_{r}$ with $I_{r}$ so that we define the inner product $A_{r}^{p}$ on $I_{r}$ by $A_{r}^{p}(x, y)=\left(d_{r}^{-1} x\right) y$. (Where there is no ambiguity, we shall denote the inner product simply by $\langle\rangle$,$) .$ Lemma 3 implies that if $m$ is odd then $\langle x, y\rangle=\langle y, x\rangle$, i.e., $\langle$,$\rangle is sym-$ metric. 
LEMmA 4. If $m$ is even, and if either $p \neq 2$ or $p=2$ and $r>1$, then $\langle$,$\rangle is symplectic, i.e., \langle x, x\rangle=0$ for $x \in I_{r}$.

Proof. It follows from Lemma 3 that $\langle x, y\rangle=-\langle y, x\rangle$, which proves Lemma 4 if $p \neq 2$. If $p=2$ and $r>1$, we recall that by [3, Theorem 5.4], if $d_{r} x=y, x \in E_{(r)}^{m}, m$ even, then $d_{r+1}\left\{x^{2}\right\}=\{x y\}$. Then by Lemma $2,\{x y\}=0$ in $E_{(r+1)}^{n}$, and since $E_{(r)}^{n}=E_{(r+1)}^{n}, x y=0$ in $E_{(r)}^{n}$, and $\langle y, y\rangle=0$.

Lemma 5. If $m$ is even and $x \in H^{m}\left(X ; Z_{2}\right)$, then $S q^{m} S q^{1} x=x S q^{1} x$ $=\left\langle S q^{1} x, S q^{1} x\right\rangle$.

Proof. By [3, Theorem 5.4], $d_{2}\left\{x^{2}\right\}=\left\{x S q^{1} x+S q^{m} S q^{1} x\right\}$. Since $d_{2}\left\{x^{2}\right\}=0$ by Lemma $2,\left\{x S q^{1} x+S q^{m} S q^{1} x\right\}=0$ in $E_{(2)}$, hence $x S q^{1} x$ $=S q^{m} S q^{1} x$.

Next we define Stiefel-Whitney classes (S-W classes) $w_{k}$ in $H^{*}\left(X ; Z_{2}\right)$ after the method of Wu (see [8]). Namely, since Poincaré duality holds in $H^{*}\left(X ; Z_{2}\right)$, the homomorphism $S q^{i}: H^{n-i}\left(X ; Z_{2}\right)$ $\rightarrow H^{n}\left(X ; Z_{2}\right)$ coincides with $u_{i} \cup$ for some $u_{i} \in H^{i}\left(X ; Z_{2}\right)$. Set $U=\sum_{i} u_{i}, S q=\sum_{j} S q^{j}$. Define $W=\sum_{k} w_{k}, w_{k} \in H^{k}\left(X ; Z_{2}\right)$ by $W=S q U$. We get immediately:

Lemma 6. $W=0$ if and only if $U=0$ if and only if $S q^{i}: H^{n-i}\left(X ; Z_{2}\right)$ $\rightarrow H^{n}\left(X ; Z_{2}\right)$ is zero for all $i$.

Proof of Theorem 1. A vector space with a symplectic inner product is even dimensional (see [2]). Then it follows from Lemma 4 and Lemma 1 that if $p$ is odd the number of summands $Z_{p} r$ in $H^{m+1}(X)$ (hence $H_{m}(X)$ by Poincaré duality) is even. This gives us alternatives (1) or (2). If $S q^{m}: I_{1} \rightarrow H^{n}\left(X ; Z_{2}\right)$ is zero $\left(I_{1}=\text { (image } S q^{1}\right)^{m+1}$ ), then from Lemma $5, A_{1}^{2}$ is symplectic and alternative (1) holds. Hence if alternative (2) holds, by Lemma 6, $W \neq 0$.

Since (image $\left.S q^{1}\right)^{n}=0$ by Lemma 2 , and $S q^{2 j+1}=S q^{1} S q^{2 j}$, it follows that only even squares reach dimension $n$ and hence $U$ is the sum of even dimensional elements. If $m=2$, then $U=u_{2}$, for $S q^{4}=0$ for dimensional reasons in $H^{*}\left(X ; Z_{2}\right)$. If alternative (2) holds in this case, then $S q^{2}: I_{1} \rightarrow H^{5}\left(X ; Z_{2}\right)$ is nonzero so that $U=u_{2} \neq 0$ and $u_{2} S q^{1} x$ $=S q^{2} S q^{1} x \neq 0$, and $u_{2} S q^{1} x=\left(S q^{1} u_{2}\right) x$ by Lemma 3 . Hence $w_{3}=S q^{1} u_{2}$ $\neq 0$.

Conversely if $m=2$ and $w_{3} \neq 0$, then $w_{3}=S q^{1} u_{2}$. From Lemma 3 and the fact that $A_{1}^{2}$ is nonsingular it follows that $u_{2} \cdot y \neq 0$ for some $y=S q^{1} x \in H^{3}\left(X ; Z_{2}\right)$. Hence $S q^{2} y=u_{2} \cdot y=S q^{2} S q^{1} x \neq 0$ and kernel $S q^{2}: I_{1} \rightarrow H^{5}\left(X ; Z_{2}\right)$ is not all of $I_{1}$, so that $\operatorname{dim}\left(\right.$ kernel $\left.S q^{2}\right)=\operatorname{dim} I_{1}-1$ (since $S q^{2}$ is a linear map into a space of dimension 1). Restricted to 
kernel $S q^{2}, A_{1}^{2}$ is symplectic by Lemma 5 , so that $\operatorname{dim} I_{1}$ is odd and alternative (2) holds. This completes the proof of Theorem 1.

Theorem 2 will follow from Theorem 1 and the following lemma:

LEMMA 7. If $w_{i}=0$ for $i<m, m$ even $>2$, then $w_{m+1}=0$.

Proof. By a result of J. F. Adams [1] relations holding among $\mathrm{S}-\mathrm{W}$ classes in differentiable manifolds, hold also for our case. If $m=2 l$, according to the formula of $\mathrm{Wu}[9],{ }^{3} S q^{l} w_{l+1}=w_{m+1}+D$, where $D$ is a product of $w_{i}$ 's for $i \leqq m$. Since $w_{i}=0$ for $i<m, D=0$ so that $S q^{l} w_{l+1}=w_{m+1}$. If $l>1$, then $l+1<2 l$ so that $w_{l+1}=0$ and hence $w_{m+1}=0$.

Proof of Theorem 2. First let us suppose $H_{m}(X)=F+T+T+Z_{2}$. Then $A_{1}^{2}$ is not symplectic and $S q^{m} S q^{1} x \neq 0$ for some $x \in H^{m}\left(X ; Z_{2}\right)$. As in the proof of the last part of Theorem $1, U=u_{m}$ and $u_{m} S q^{1} x$ $=S q^{m} S q^{1} x \neq 0$. But $u_{m} S q^{1} x=\left(S q^{1} u_{m}\right) x$, so that $S q^{1} u_{m} \neq 0$ and $W=u_{m}$ $+S q^{1} u_{m}$ so that $w_{m}=u_{m}$ and $w_{m+1}=S q^{1} u_{m} \neq 0$. Hence $m=2$ by Lemma 7.

The converse follows immediately from Theorem 1.

Example of differentiable manifolds which satisfy the hypotheses of Theorem 2 are described in [6].

\section{BIBLIOGRAPHY}

1. J. F. Adams, On the formuli of Thom and Wu, Proc. London Math. Soc. 11 (1961), 741-752.

2. E. Artin, Geometric algebra, Interscience, New York, 1957.

3. W. Browder, Torsion in H-spaces, Ann. of Math. (2) 74 (1961), 24-51.

4. M. Rueff, Beitrage zur Untersuchung der Abbildungen von Mannigfaltigen, Compositio Math. 6 (1938), 161-202.

5. H. Seifert, Verschlingungsinvarianten, S.-B. Preuss. Akad. Wiss. 16 (1933), 811-828.

6. S. Smale, On the structure of 5-manifolds, Ann. of Math. (2) 75 (1962), 38-46.

7. C. T. C. Wall, Killing the middle homotopy groups of odd dimensional manifolds, Trans. Amer. Math. Soc. 103 (1962), 421-433.

8. W. T. Wu, Classes caracteristiques et i-carrés d'une variette, C. R. Acad. Sci. Paris 230 (1950), 508-511.

9. - Les i-carrés dans une variêté grassmannienne, C. R. Acad. Sci. Paris 230 (1950), 918-920.

\section{CoRnell University}

${ }^{3}$ This fact does not require the full strength of $\mathrm{Wu}$ 's formula, but can be seen very easily by considering the cohomology suspension $\sigma^{*}: H^{*}\left(B_{0} ; Z_{2}\right) \rightarrow H^{*}\left(0 ; Z_{2}\right)$. It is well known that $\sigma^{*}\left(w_{i}\right)=h_{i-1}$ where $\left\{h_{m}\right\}$ are a simple system of generators for $H^{*}\left(0 ; Z_{2}\right)$, the $w_{i}$ are a set of generators for $H^{*}\left(B_{0} ; Z_{2}\right)$ and kernel $\sigma^{*}=$ decomposable elements. Then the result we need follows from the relation $S q^{k} h_{k}=h^{2}{ }_{k}=h_{2 k}$ in $H^{*}\left(0 ; Z_{2}\right)$. 